\title{
Exact Dynamics of an Infinite-Atom Dicke Maser Model
}

\author{
E. B. Davies \\ Mathematical Institute, Oxford, U.K.
}

Received March 9, 1973

\begin{abstract}
We study the dynamics of the Dicke maser model in the limit as the number of atoms becomes infinite and the coupling constant between the atoms and the radiation field goes to zero. We find that the limiting Hamiltonian is integrable and obtain an explicit closed form for the unitary time evolution operators. As a corollary we show that in the limit the radiation emitted by the model is coherent in the sense by Glauber.
\end{abstract}

\section{$\S 1$. Formulation of the Problem}

We study the radiation emitted by a large system of atoms in a superradiant state. We make the electric dipole approximation for each atom, supposing that the overall dimensions of the system are small compared to the wavelength of the emitted radiation. The radiation is supposed to consist of photons, but for notational simplicity we let the emitted particles be bosons of unspecified nature.

We set up the Hilbert space and Hamiltonian in the standard manner $[6,8,15]$. Each atom is described by a two-dimensional space $\mathbb{C}^{2}$, and the system of $n$ atoms by $\otimes{ }^{n} \mathbb{C}^{2}$. For the $r$ th atom we introduce the spin operators $J^{(r)}$ acting on the $r$ th component of $\otimes{ }^{n} \mathbb{C}^{2}$ and satisfying the commutation relations

$$
\begin{gathered}
{\left[J_{1}^{(r)}, J_{2}^{(r)}\right]=i J_{3}^{(r)} ; \quad\left[J_{2}^{(r)}, J_{3}^{(r)}\right]=i J_{1}^{(r)} ; \quad\left[J_{3}^{(r)}, J_{1}^{(r)}\right]=i J_{2}^{(r)} ;} \\
J_{+}^{(r)}=J_{1}^{(r)}+i J_{2}^{(r)} ; \quad J_{-}^{(r)}=J_{1}^{(r)}-i J_{2}^{(r)} ; \quad\left[J_{i}^{(r)}, J_{j}^{(s)}\right]=0 \quad \text { if } \quad r \neq s .
\end{gathered}
$$

The single particle space for the emitted radiation is denoted by $\mathscr{H}$ and the quantised radiation field is the boson Fock space $\mathscr{F}$ over $\mathscr{H}$ :

$$
\mathscr{F}=\mathbb{C} \oplus \mathscr{H} \oplus\left\{\otimes_{\text {sym }}^{2} \mathscr{H}\right\} \oplus\left\{\otimes_{\text {sym }}^{3} \mathscr{H}\right\} \oplus \cdots .
$$

For any $f, g \in \mathscr{H}$ we have smeared creation and annihilation operators $a^{*}(f)$ and $a(g)$ on $\mathscr{F}$ with commutation relations

$$
\begin{gathered}
a(g) a^{*}(f)-a^{*}(f) a(g)=\langle f, g\rangle 1, \\
{[a(g), a(f)]=0 .}
\end{gathered}
$$


We let $S \geqq 0$ denote the single particle Hamiltonian on $\mathscr{H}$ and $H_{0}$ the corresponding free Hamiltonian on $\mathscr{F}$.

The Hilbert space for the composite system is

$$
\left\{\otimes \mathbb{C}^{n}\right\} \otimes \mathscr{F}
$$

and the Hamiltonian for the Dicke maser model is

$$
H_{n}=\sum_{r=1}^{n} \omega J_{3}^{(r)}+H_{0}+\frac{\lambda}{n} \sum_{r=1}^{n}\left\{J_{-}^{(r)} a^{*}(b)+J_{+}^{(r)} a(b)\right\} .
$$

Here $b \in \mathscr{H}$ gives the mode of the radiation field to which the atoms are supposed to be coupled; $b$ is not supposed to be an eigenvector of $S$. The coupling constant is taken as $\lambda / n$ because we desire a finite rate of radiation in the limit $n \rightarrow \infty$, and it is expected that the rate of radiation is proportional to the square of the number of atoms [8, p. 221]. This contrasts with [10] where the coupling constant is taken to be $\lambda / \sqrt{n}$ for thermodynamic equilibrium reasons. In the above equations, and below, we use expressions such as $H_{0}$ to denote both the operator on $\mathscr{F}$ and the operator $1 \otimes H_{0}$ on $\left\{\otimes \mathbb{C}^{n}\right\} \otimes \mathscr{F}$.

The operator $H_{n}$ is a self-adjoint operator with the same domain as $H_{0}$. This is proved by noting that the third term in Eq. (1.4) is relatively bounded perturbation of $H_{0}$ with arbitrarily small bound [13, p. 187].

We suppose that at time $t=0$ the state of the atomic system is given by the density matrix $\varrho_{n}$ and that the state of the radiation field is given by the positive normalised trace class operator $\varrho$ on $\mathscr{F}$. Since we are interested only in the state of the radiation field at time $t$ we introduce the map

$$
M_{n}: \mathscr{T}_{s}\left(\left\{\otimes{ }^{n} \mathbb{C}^{2}\right\} \otimes \mathscr{F}\right) \rightarrow \mathscr{T}_{s}(\mathscr{F})
$$

which is obtained by averaging out the atomic state. Here $\mathscr{T}_{s}(\mathscr{F})$ is the space of self-adjoint trace class operators on $\mathscr{F} . M$ is a positive linear trace-preserving map given explicitly by

$$
\operatorname{tr}[M(\varrho) A]=\operatorname{tr}[\varrho(1 \otimes A)]
$$

where $A$ is an arbitrary bounded operator on $\mathscr{F}$.

The state of the radiation field at time $t$ is then given by

$$
T_{n, t}(\varrho)=M_{n}\left[e^{-i H_{n} t}\left\{\varrho_{n} \otimes \varrho\right\} e^{i H_{n} t}\right]
$$

for $t \geqq 0$ and arbitrary $\varrho \in \mathscr{T}_{s}(\mathscr{F})$. The limiting dynamics of the field is given by

$$
T_{t}(\varrho)=\lim _{n \rightarrow \infty} T_{n, t}(\varrho) .
$$

The main result of the paper is to show that the limit does exist under suitable assumptions, and that it can be written as an exact closed form. 
Before proceeding we draw the reader's attention to two interesting papers on related problems which have recently appeared $[7,10]$. For an account of the Dicke maser model with a finite number of atoms see $[6,18,21]$.

\section{§ 2. Details of the Infinite Atom Limit}

The form of the limit in Eq. (1.8) will depend on the initial states $\varrho_{n}$ of the system of atoms. To investigate this we define

so that

$$
J_{3}=\sum_{r=1}^{n} J_{3}^{(r)} ; \quad J_{ \pm}=\sum_{r=1}^{n} J_{ \pm}^{(r)}
$$

$$
\left[J_{3}, J_{ \pm}\right]= \pm J_{ \pm} ; \quad\left[J_{+}, J_{-}\right]=2 J_{3}
$$

and the Hamiltonian $H_{n}$ can be rewritten as

Writing

$$
H_{n}=\omega J_{3}+H_{0}+\frac{\lambda}{n}\left\{J_{-} a^{*}(b)+J_{+} a(b)\right\} \text {. }
$$

it is clear that

$$
J^{2}=J_{1}^{2}+J_{2}^{2}+J_{3}^{2}=J_{-} J_{+}+J_{3}\left(J_{3}+1\right)
$$

$$
\left[J^{2}, H_{n}\right]=0
$$

which suggests reducing the problem with respect to the representation of the Lie algebra $s l(2, \mathbb{C})$ generated by $J_{3}, J_{ \pm} . s l(2, \mathbb{C})$ has exactly one representation $\pi_{m}$ of each dimension $m$ and in the representation $\pi_{m}, J^{2}$ has the constant value $\left(m^{2}-1\right) / 4$. It follows from standard formulae [16] that

$$
\otimes^{n} \pi_{2}=\sum_{m=1}^{n+1} a_{n, m} \pi_{m}
$$

where the integers $a_{n, m}$ are multiplicities and $a_{n, n+1}=1$. Therefore in $\otimes \mathbb{C}^{2}, J^{2}$ takes values ranging from zero to $n(n+2) / 4$.

If $\sigma$ is an arbitrary density matrix on $\otimes^{n} \mathbb{C}^{2}$ we now define its (root mean square) cooperation number $\mu$ by

$$
\operatorname{tr}\left[\sigma J^{2}\right]=\mu n(\mu n+2) / 4
$$

so that $0 \leqq \mu \leqq 1$ (compare [6]). We also define the proportion $\gamma$ of atoms in their excited state by

$$
\operatorname{tr}\left[\sigma J_{3}\right]=\gamma n-n / 2
$$

so that also $0 \leqq \gamma \leqq 1$. We assume that $\mu$ and $\gamma$ have limiting values for the sequence of states $\varrho_{n}$, and ensure this in the simplest possible way. Namely we assume that $\varrho_{n}$ is a vector state

$$
\varrho_{n}=\xi_{n} \otimes \bar{\xi}_{n}
$$


where

$$
J^{2} \xi_{n}=\frac{1}{4} \mu_{n} n\left(\mu_{n} n+2\right) \xi_{n}, \quad \lim _{n \rightarrow \infty} \mu_{n}=\mu
$$

and

$$
J_{3} \xi_{n}=\left(\gamma_{n} n-n / 2\right) \xi_{n}, \quad \lim _{n \rightarrow \infty} \gamma_{n}=\gamma \text {. }
$$

If $m=\mu_{n} n$ then the subspace $L_{n}$ of $\otimes^{n} \mathbb{C}^{2}$ generated by $\xi_{n}$ and $\operatorname{sl}(2, \mathbb{C})$ is $m$-dimensional and has a basis $\varphi_{1}, \ldots, \varphi_{m}$ where

$$
\begin{aligned}
& J_{+} \varphi_{s}=\sqrt{s(m-s)} \varphi_{s+1}, \\
& J_{-} \varphi_{s}=\sqrt{(s-1)(m-s+1)} \varphi_{s-1}, \\
& J_{3} \varphi_{s}=\left(s-m / 2-\frac{1}{2}\right) \varphi_{s} .
\end{aligned}
$$

Moreover by Eq. (2.9)

where

$$
\xi_{n}=\varphi_{p}
$$

Therefore

$$
\gamma_{n} n-n / 2=p-m / 2-\frac{1}{2} \text {. }
$$

$$
p / m-\frac{1}{2}=\frac{1}{2 m}+\frac{\gamma_{n}}{\mu_{n}}-\frac{1}{2 \mu_{n}}
$$

and since $1 \leqq p \leqq m$, letting $n \rightarrow \infty$ yields

$$
\left|\gamma-\frac{1}{2}\right| \leqq \frac{1}{2} \mu
$$

a restriction on $\gamma$ and $\mu$ which is not immediately apparent from Eqs. (2.8) and (2.9). For the sake of eliminating some degenerate cases, we assume from now on that

$$
0<\mu \leqq 1, \quad\left|\gamma-\frac{1}{2}\right|<\frac{1}{2} \mu .
$$

For future reference we note that the integers $m$ and $p$ depend on $n$ and that the limiting behaviour is

$$
\lim _{n \rightarrow \infty}(m / n)=\mu, \quad \lim _{n \rightarrow \infty}(p / n)=\gamma-\frac{1}{2}+\frac{\mu}{2} .
$$

Since the subspace $L_{n}$ of $\otimes{ }^{n} \mathbb{C}^{2}$ is invariant under $\operatorname{sl}(2, \mathbb{C})$, the subspace $L_{n} \otimes \mathscr{F}$ of $\left\{\otimes n \mathbb{C}^{2}\right\} \otimes \mathscr{F}$ is invariant under the Hamiltonian $H_{n}$ and therefore under the unitary group $e^{i H_{n} t}$. We may then take the Hamiltonian $H_{n}$ of Eq. (2.3) as being defined on $L_{n} \otimes \mathscr{F}$ since the limiting behaviour of Eq. (1.8) refers only to the second term of the tensor product. The next step in analysing the sequence of Hamiltonians consists of transferring them to a Hilbert space independent of $n$. For this purpose we introduce the space $l^{2}(\mathbb{Z})$ of square summable sequences and the orthonormal basis $\left\{e_{n}\right\}_{n=-\infty}^{\infty}$ where $e_{n}$ is the sequence whose $m$ th term is $\delta_{m n}$. We let

$$
A_{n}: L_{n} \rightarrow l^{2}(\mathbb{Z})
$$

be the isometric embeddings given by

$$
A_{n}\left(\sum_{r=1}^{m} \alpha_{r} \varphi_{r}\right)=\sum_{r=1}^{m} \alpha_{r} e_{r-p}
$$


so that

$$
A_{n} \xi_{n}=e_{0}
$$

independently of $n$. We also use $A_{n}$ to denote the corresponding isometric embedding

$$
A_{n}: L_{n} \otimes \mathscr{F} \rightarrow l^{2}(\mathbb{Z}) \otimes \mathscr{F} \equiv \mathscr{K}
$$

and define the Hamiltonian $K_{n}$ on $\mathscr{K}$ by

$$
K_{n}=A_{n} H_{n} A_{n}^{*}
$$

so that $K_{n}$ is unitarily equivalent to $H_{n}$ on the subspace $A_{n}\left(L_{n}\right) \otimes \mathscr{F}$ of $\mathscr{K}$ and equal to zero on the orthogonal complement of this subspace. If $\varrho_{0}=e_{0} \otimes \bar{e}_{0}$ then it is fairly obvious from Eq. (1.7) and the subsequent calculations that

$$
T_{n, t}(\varrho)=M\left[e^{-i K_{n} t}\left\{\varrho_{0} \otimes \varrho\right\} e^{i K_{n} t}\right] .
$$

The operation $M$ denotes taking the partial trace with respect to $l^{2}(\mathbb{Z})$.

We give an explicit expression for the self-adjoint operator $K_{n}$. Let $B_{3, n}, B_{ \pm, n}$ be the operators on $l^{2}(\mathbb{Z})$ defined by

$$
\begin{aligned}
& B_{+, n} e_{s}=n^{-1}\{(s+p)(m-s-p)\}^{\frac{1}{2}} e_{s+1}, \\
& B_{-, n} e_{s}=n^{-1}\{(s+p-1)(m-s-p+1)\}^{\frac{1}{2}} e_{s-1}, \\
& B_{3, n} e_{s}=\{s+p-m / 2-1 / 2\} e_{s}
\end{aligned}
$$

if $1-p \leqq s \leqq m-p$ and

$$
B_{\cdot, n} e_{s}=0
$$

otherwise. It follows from Eq. (2.22) that

$$
K_{n}=\omega B_{3, n}+H_{0}+\lambda\left\{B_{-, n} a^{*}(b)+B_{+, n} a(b)\right\} .
$$

Note that the factor $n^{-1}$ in the coupling constant has now been absorbed into the operators $B_{ \pm, n}$. It is immediate from their definitions and Eq. (2.17) that $B_{ \pm, n}$ converge strongly as $n \rightarrow \infty$ to the bounded operators

where

$$
B_{ \pm} e_{s}=\beta e_{s \pm 1}
$$

$$
\beta=\left\{\left(\gamma-\frac{1}{2}+\frac{\mu}{2}\right)\left(-\gamma+\frac{1}{2}+\frac{\mu}{2}\right)\right\}^{\frac{1}{2}} .
$$

The proof of this is facilitated by use of the easily proved bound

$$
\left\|B_{ \pm, n}\right\| \leqq m / 2 n \leqq \mu / 2+1
$$

for all large enough $n$. The situation with $B_{3, n}$ is more complicated since one has to subtract a constant depending on $n$ to obtain convergence. If $B_{3}$ is the unbounded operator defined by

$$
B_{3} e_{s}=s e_{s}
$$


then

$$
\lim _{n \rightarrow \infty}\left\{B_{3, n}-\left(p-\frac{m}{2}-\frac{1}{2}\right) 1\right\}=B_{3}
$$

where the convergence is taken in the strong generalised sense [13]. The need to subtract this divergent constant is acceptable since addition of a constant to $K_{n}$ makes no difference to $T_{n, t}$ in Eq. (2.23).

\section{§ 3. Integration of the Limiting Hamiltonian}

It is clear from the above formulae that as $n \rightarrow \infty, K_{n}$ converges in some sense to the operator given formally by

$$
K=\omega B_{3}+H_{0}+\lambda\left\{B_{-} a^{*}(b)+B_{+} a(b)\right\} .
$$

It turns out that there is an exact closed form for the unitary group $e^{i K t}$, the ultimate reason for this being that in the limit the operators $J$ have been replaced by the operators $B$ which satisfy the simpler commutation relations

$$
\left[B_{3}, B_{ \pm}\right]= \pm B_{ \pm} ; \quad\left[B_{+}, B_{-}\right]=0 .
$$

We have, in fact, a particular case of the phenomenon of contraction of Lie algebras $[11,12]$.

We carry out all calculations in the Fourier transform representation. We let

$$
U: L^{2}(-\pi, \pi) \rightarrow l^{2}(\mathbb{Z})
$$

be the unitary isomorphism

$$
\{U f\}_{n}=\frac{1}{\sqrt{2 \pi}} \int_{-\pi}^{\pi} f(\theta) e^{-i n \theta} d \theta
$$

and, identifying $L^{2}(-\pi, \pi) \otimes \mathscr{F}$ with the Hilbert space $L^{2}[(-\pi, \pi), \mathscr{F}] \equiv \hat{\mathscr{K}}$ of all square integrable $\mathscr{F}$-valued functions on $(-\pi, \pi)$, also let $U$ denote the corresponding unitary isomorphism

The operator

$$
U: \hat{\mathscr{K}} \rightarrow \mathscr{K} .
$$

is given formally by

$$
\hat{K}=U^{*} K U
$$

$$
\begin{aligned}
(\hat{K} \psi)(\theta) & =-i \omega \frac{\partial \psi}{\partial \theta}+H_{0} \psi(\theta) \\
& =\lambda \beta\left\{e^{-i \theta} a^{*}(b)+e^{+i \theta} a(b)\right\} \psi(\theta) .
\end{aligned}
$$

Defining the smeared field operators $\Phi$ by

for any $f \in \mathscr{H}$, we get

$$
\Phi(f)=2^{-\frac{1}{2}}\left\{a^{*}(f)+a(f)\right\}
$$

$$
(\hat{K} \psi)(\theta)=-i \omega \frac{\partial \psi}{\partial \theta}+H_{0} \psi(\theta)+2^{\frac{1}{2}} \lambda \beta \Phi\left(e^{-i \theta} b\right) \psi(\theta) .
$$


Instead of defining a domain of essential self-adjointness of $\hat{K}$, we proceed to a direct construction of the unitary group $\exp \{-i \hat{K} t\}$ and then return to prove that $\hat{K}$ is indeed its infinitesimal generator.

We recall from $[1,4,19]$ that for any $f \in \mathscr{H}$ there is a unitary operator $W(f)$ on $\mathscr{F}$ given by

$$
W(f)=\exp [i \Phi(f)]
$$

and satisfying the canonical commutation relations

$$
W(f) W(g)=W(f+g) \exp [i \operatorname{Im}\langle f, g\rangle / 2] .
$$

Moreover the commutation relation between $W$ and $e^{i H_{0} t}$ are

$$
e^{i H_{0} t} W(f) e^{-i H_{0} t}=W\left(e^{i S t} f\right)
$$

where we defined $S$ as the single particle Hamiltonian on $\mathscr{H}$. We define a class of one-parameter unitary groups on $\hat{\mathscr{K}}$ by means of cocycle equations, a technique developed extensively in [17].

Theorem 3.1. The equation

$$
\left(V_{t} \psi\right)(\theta)=e^{i \alpha(\theta . t)} W\{f(\theta, t)\} e^{-i H_{0} t} \psi(\theta-\omega t)
$$

defines a one-parameter unitary group on $\hat{\mathscr{K}}$ if and only if $\alpha$ and $f$ satisfy the cocycle equations

$$
\begin{gathered}
f(\theta, s+t)=f(\theta, s)+e^{-i S s} f(\theta-\omega s, t), \\
\alpha(\theta, s+t)=\alpha(\theta, s)+\alpha(\theta-\omega s, t)+\operatorname{Im}\left\langle f(\theta, s), e^{-i S s} f(\theta-\omega s, t)\right\rangle / 2
\end{gathered}
$$

almost everywhere. All solutions of these equations satisfy

$$
f(\theta, 0)=0, \quad \alpha(\theta, 0)=0 .
$$

Proof. It is clear that for all real $t$, Eq. (3.12) defines a unitary operator $V_{t}$ on $\mathscr{K}$, so we only have to verify the group equation.

$$
\begin{aligned}
\left(V_{s} V_{t} \psi\right)(\theta)= & e^{i \alpha(\theta, s)} W\{f(\theta, s)\} e^{-i H_{0} s}\left(V_{t} \psi\right)(\theta-\omega s) \\
= & e^{i \alpha(\theta, s)} W\{f(\theta, s)\} e^{-i H_{0} s} \\
& \cdot e^{i \alpha(\theta-\omega s . t)} W\{f(\theta-\omega s, t)\} e^{-i H_{0} t} \psi(\theta-\omega s-\omega t) \\
= & e^{i \alpha(\theta, s)+i \alpha(\theta-\omega s, t)} W\{f(\theta, s)\} W\left\{e^{-i S s} f(\theta-\omega s, t)\right\} \\
& \cdot e^{-i H_{0}(s+t)} \psi(\theta-\omega(s+t)) \\
= & \exp \left[i \alpha(\theta, s)+i \alpha(\theta-\omega s, t)+i \operatorname{Im}\left\langle f(\theta, s), e^{-i S s} f(\theta-\omega s, t)\right\rangle / 2\right] \\
& \cdot W\left\{f(\theta, s)+e^{-i S s} f(\theta-\omega s, t)\right\} e^{-i H_{0}(s+t)} \psi(\theta-\omega(s+t))
\end{aligned}
$$

from which Eqs. (3.13) and (3.14) follow. Putting $t=0$ in these equations yields Eq. (3.15).

We transform the cocycle equations into more comprehensible differential equations. 
Theorem 3.2. Let $f, \alpha$ be solutions of the cocycle equations with continuous first partial derivatives and let

$$
g(\theta)=\frac{\partial f}{\partial s}(\theta, 0), \quad \beta(\theta)=\frac{\partial \alpha}{\partial s}(\theta, 0) .
$$

Then $f$ and $\alpha$ satisfy the differential equations

$$
\begin{gathered}
\frac{\partial f}{\partial s}(\theta, s)=e^{-i s s} g(\theta-\omega s), \\
\frac{\partial \alpha}{\partial s}(\theta, s)=\beta(\theta-\omega s)+\operatorname{Im}\left\langle f(\theta, s), e^{-i S s} g(\theta-\omega s)\right\rangle / 2 .
\end{gathered}
$$

Conversely given $g$ and $\beta$, these differential equations have unique solutions satisfying the initial conditions

$$
f(\theta, 0)=0, \quad \alpha(\theta, 0)=0
$$

and the solutions satisfy the cocycle equations.

Proof. Using Eq. (3.15) we may rewrite Eq. (3.13) as

$$
\frac{f(\theta, s+t)-f(\theta, s)}{t}=e^{-i s s} \frac{f(\theta-\omega s, t)-f(\theta-\omega s, 0)}{t}
$$

which on letting $t \rightarrow 0$ becomes Eq. (3.18). Similarly Eq. (3.14) may be rewritten as

$$
\begin{aligned}
& \frac{\alpha(\theta, s+t)-\alpha(\theta, s)}{t}=\frac{\alpha(\theta-\omega s, t)-\alpha(\theta-\omega s, 0)}{t} \\
& +\frac{1}{2} \operatorname{Im}\left\langle f(\theta, s), e^{-i s s} \frac{f(\theta-\omega s, t)-f(\theta-\omega s, 0)}{t}\right\rangle
\end{aligned}
$$

which on letting $t \rightarrow 0$ becomes Eq. (3.19).

Conversely given $g$ the differential equation (3.18) has a unique solution with the said initial conditions and this solution is

Therefore

$$
f(\theta, s)=\int_{u=0}^{s} e^{-i S u} g(\theta-\omega u) d u .
$$

$$
\begin{aligned}
f(\theta, s) & +e^{-i S s} f(\theta-\omega s, t) \\
= & \int_{u=0}^{s} e^{-i S u} g(\theta-\omega u) d u+\int_{v=0}^{t} e^{-i S(s+v)} g(\theta-\omega s-\omega v) d v \\
= & \int_{u=0}^{s} e^{-i S u} g(\theta-\omega u) d u+\int_{u=s}^{s+t} e^{-i S u} g(\theta-\omega u) d u \\
= & f(\theta, s+t)
\end{aligned}
$$

so $f$ satisfies the cocycle equation (3.13).

Similarly the solution of the differential equation (3.19) is

$$
\alpha(\theta, s)=\alpha_{1}(\theta, s)+\alpha_{2}(\theta, s)
$$


where

and

Now

$$
\alpha_{2}(\theta, s)=\frac{1}{2} \int_{u=0}^{s} \int_{v=0}^{u} \operatorname{Im}\left\langle e^{-i S v} g(\theta-\omega v), e^{-i S u} g(\theta-\omega u)\right\rangle d v d u
$$

$$
\begin{aligned}
\alpha_{1}(\theta, s) & +\alpha_{1}(\theta-\omega s, t) \\
& =\int_{u=0}^{s} \beta(\theta-\omega u) d u+\int_{v=0}^{t} \beta(\theta-\omega s-\omega v) d v \\
& =\alpha_{1}(\theta, s+t)
\end{aligned}
$$

so $\alpha$ satisfies Eq. (3.14) provided $\alpha_{2}$ satisfies it.

$$
\begin{aligned}
& \alpha_{2}(\theta, s)+\alpha_{2}(\theta-\omega s, t)+\frac{1}{2} \operatorname{Im}\left\langle f(\theta, s), e^{-i S s} f(\theta-\omega s, t)\right\rangle \\
& =\frac{1}{2} \int_{u=0}^{s} \int_{v=0}^{u} \operatorname{Im}\left\langle e^{-i S v} g(\theta-\omega v), e^{-i S u} g(\theta-\omega u)\right\rangle d v d u \\
& +\frac{1}{2} \int_{u=0}^{t} \int_{v=0}^{u} \operatorname{Im}\left\langle e^{-i S v} g(\theta-\omega s-\omega v), e^{-i S u} g(\theta-\omega s-\omega u)\right\rangle d v d u \\
& +\frac{1}{2} \int_{v=0}^{s} \int_{u=0}^{t} \operatorname{Im}\left\langle e^{-i S v} g(\theta-\omega v), e^{-i S s} e^{-i S u} g(\theta-\omega s-\omega u)\right\rangle d v d u \\
& =\frac{1}{2} \int_{x=0}^{s} \int_{y=0}^{x} \operatorname{Im}\left\langle e^{-i S y} g(\theta-\omega y), e^{-i S x} g(\theta-\omega x)\right\rangle d y d x \\
& +\frac{1}{2} \int_{x=s}^{t} \int_{y=s}^{x} \operatorname{Im}\left\langle e^{-i S y} g(\theta-\omega y), e^{-i S x} g(\theta-\omega x)\right\rangle d y d x \\
& +\frac{1}{2} \int_{y=0}^{s} \int_{x=s}^{s+t} \operatorname{Im}\left\langle e^{-i S y} g(\theta-\omega y), e^{-i S x} g(\theta-\omega x)\right\rangle d y d x \\
& =\alpha_{2}(\theta, s+t)
\end{aligned}
$$

which completes the proof of the theorem.

From now on we suppose that $f, \alpha$ are given cocycles with continuous first partial derivatives, and that $f(\theta, t) \in \mathscr{D}(S)$ for all $\theta, t$. We define a self-adjoint operator $\hat{K}$ by

$$
V_{t}=\exp [-i \hat{K} t]
$$

where $V_{t}$ is given by Eq. (3.12). In order to find an explicit expression for $\hat{K}$ we introduce some suitable domains. For any $\alpha>0$ we define

$$
\mathscr{F}_{\alpha}=\left\{\psi \in \mathscr{F}:\|\psi\|_{\alpha} \equiv\left\|e^{\alpha N} \psi\right\|<\infty\right\}
$$

where $N$ is the number operator on $\mathscr{F}$ and

$$
\mathscr{F}_{\infty}=\bigcap_{\alpha} \mathscr{F}_{\alpha},
$$

giving $\mathscr{F}_{\infty}$ the Frechet space topology induced by all the norms $\|\cdot\|_{\alpha}$. The operators $W(f)$ leave $\mathscr{F}_{\infty}$ invariant for all $f \in \mathscr{H}$, as do the operators 
$\Phi(f)$. On this domain the map $f \rightarrow W(f)$ is strongly differentiable in the following sense.

Proposition 3.3. Let $h(t)$ be a differentiable $\mathscr{H}$-valued function and let $\psi \in \mathscr{F}_{\infty}$. Then $t \rightarrow W\{h(t)\} \psi$ is a differentiable map from $\mathbb{R}$ into $\mathscr{F}_{\infty}$ and

$$
\frac{d}{d t} W\{h(t)\} \psi=i\left[-\operatorname{Im}\left\langle h^{\prime}(t), h(t)\right\rangle / 2+\Phi\left\{h^{\prime}(t)\right\}\right] W\{h(t)\} \psi .
$$

The proof of this proposition follows in a straightforward manner from well-known results about the Fock space representation of the CCR's $[1,2,19]$.

Lemma 3.4. Let $\mathscr{D}_{1} \subseteq \hat{\mathscr{K}}$ be the space of all continuously differentiable periodic functions from $[-\pi, \pi]$ to $\mathscr{F}_{\infty}$. Then $\mathscr{D}_{1}$ is dense in $\hat{\mathscr{K}}$ and invariant under $\exp [-i \hat{K} t]$ for all $t \in \mathbb{R}$.

Proof. We show that $\mathscr{D}_{1}$ is invariant under each of the separate components of $V_{t}$ in Eq. (3.12). If $\psi \in \mathscr{D}_{1}$ then

$$
\begin{aligned}
& \left\|\frac{\partial}{\partial \theta} e^{i H_{0} t} \psi(\theta)\right\|_{\alpha}=\left\|e^{i H_{0} t} \frac{\partial \psi}{\partial \theta}\right\|_{\alpha}=\left\|\frac{\partial \psi}{\partial \theta}\right\|_{\alpha}<\infty, \\
& \left\|\frac{\partial}{\partial \theta}\{\psi(\theta+\omega t)\}\right\|_{\alpha}=\left\|\frac{\partial \psi}{\partial \theta}(\theta+\omega t)\right\|_{\alpha}<\infty, \\
& \left\|\frac{\partial}{\partial \theta} W\{f(\theta, t)\} \psi(\theta)\right\|_{\alpha} \leqq W\{f(\theta, t)\} \frac{\partial \psi}{\partial \theta} \|_{\alpha} \\
& +\left\|\left[-\operatorname{Im}\left\langle\frac{\partial f}{\partial \theta}, f\right\rangle / 2+\Phi\left\{\frac{\partial f}{\partial \theta}\right\}\right] W\{f\} \psi(\theta)\right\|_{\alpha}<\infty .
\end{aligned}
$$

The lemma follows immediately from these estimates.

Lemma 3.5. The subspace $\mathscr{D}_{2}=\mathscr{D}_{1} \cap \mathscr{D}\left(H_{0}\right)$ of $\hat{\mathscr{K}}$ is dense and in variant under $\exp [-i \hat{K} t]$ for all $t \in \mathbb{R}$.

Proof. The invariance of this domain under $e^{-i H_{0} t}$ and under $\psi(\theta)$ $\rightarrow \psi(\theta-\omega t)$ is obvious, so we only need to show that if $\psi \in \mathscr{D}_{2}$ then $W\{f(\theta, t)\} \psi(\theta) \in \mathscr{D}\left(H_{0}\right)$ for all $\theta \in[-\pi, \pi]$.

$$
\begin{aligned}
i H_{0} W\{f(\theta, t)\} \psi(\theta)=\lim _{s \rightarrow 0} s^{-1}\left[e^{i H_{0} s}-1\right] W\{f(\theta, t)\} \psi(\theta) \\
\quad=\lim _{s \rightarrow 0} s^{-1}\left[W\left\{e^{i S s} f(\theta, t)\right\} e^{i H_{0} s} \psi(\theta)-W\{f(\theta, t)\} \psi(\theta)\right] \\
\quad=\lim _{s \rightarrow 0} W\left\{e^{i S s} f(\theta, t)\right\} s^{-1}\left[e^{i H_{0} s} \psi(\theta)-\psi(\theta)\right] \\
\quad+\lim _{s \rightarrow 0} s^{-1}\left[W\left\{e^{i S s} f(\theta, t)\right\}-W\{f(\theta, t)\}\right] \psi(\theta) \\
\quad=W\{f(\theta, t)\} i H_{0} \psi(\theta) \\
\quad+i[-\operatorname{Im}\langle i S f(\theta, t), f(\theta, t)\rangle+\Phi\{i S f(\theta, t)\}] W\{f(\theta, t)\} \psi(\theta)
\end{aligned}
$$

by Proposition (3.3). 
Lemma 3.6. Let $\mathscr{L}$ be a dense subspace of a Hilbert space $\mathscr{V}$ and $A(t), B(t)$ two uniformly bounded families of operators such that $\mathscr{L}$ is invariant for both families and $t \rightarrow A(t) \psi$ and $f \rightarrow B(t) \psi$ are differentiable for all $\psi \in \mathscr{L}$. Then $t \rightarrow A(t) B(t) \psi$ is differentiable for all $\psi \in \mathscr{L}$ and

$$
\frac{d}{d t}\{A(t) B(t) \psi\}=\frac{d A}{d t} B(t) \psi+A(t) \frac{d B}{d t} \psi .
$$

Proof. Since $A(t)$ and $B(t)$ are uniformly bounded and strongly differentiable on $\mathscr{L}$ they are strongly continuous on $\mathscr{V}$. Supposing $\psi \in \mathscr{L}$ and $\|A(t)\| \leqq k,\|B(t)\| \leqq k$ for all $t$

$$
\begin{aligned}
\lim _{s \rightarrow 0} \| & S^{-1}[A(t+s) B(t+s) \psi-A(t) B(t) \psi]-\frac{d A}{d t} B(t) \psi-A(t) \frac{d B}{d t} \psi \| \\
= & \lim _{s \rightarrow 0} \| s^{-1}[A(t+s)-A(t)][B(t+s)-B(t)] \psi \\
& +s^{-1}[A(t+s)-A(t)] B(t) \psi+s^{-1} A(t)[B(t+s)-B(t)] \psi \\
& -\frac{d A}{d t} B(t) \psi-A(t) \frac{d B}{d t} \psi \| \\
\leqq & \lim _{s \rightarrow 0}\left[\left\|\{A(t+s)-A(t)\}\left[s^{-1}\{B(t+s)-B(t)\}-\frac{d B}{d t}\right] \psi\right\|\right. \\
& +\left\|\{A(t+s)-A(t)\} \frac{d B}{d t} \psi\right\| \\
& +\left\|\left[s^{-1}\{A(t+s)-A(t)\}-\frac{d A}{d t}\right] B(t) \psi\right\| \\
& \left.+\left\|A(t)\left[s^{-1}\{B(t+s)-B(t)\}-\frac{d B}{d t}\right] \psi\right\|\right] \\
\leqq & \lim _{s \rightarrow 0}\left[2 k\left\|\left[s^{-1}\{B(t+s)-B(t)\}-\frac{d B}{d t}\right] \psi\right\|\right. \\
& +\left\|\{A(t+s)-A(t)\}\left(\frac{d B}{d t} \psi\right)\right\| \\
& +\left\|\left[s^{-1}\{A(t+s)-A(t)\}-\frac{d A}{d t}\right](B(t) \psi)\right\| \\
& \left.+k\left\|\left[s^{-1}\{B(t+s)-B(t)\}-\frac{d B}{d t}\right] \psi\right\|\right] \\
= & 0
\end{aligned}
$$

which proves the lemma. 
Lemma 3.7. The operator $\hat{K}$ is essentially self-adjoint on $\mathscr{D}_{2}$ and is given on this domain by

$$
(\hat{K} \psi)(\theta)=-i \omega \frac{\partial \psi}{\partial \theta}+H_{0} \psi(\theta)-\Phi\{g(\theta)\} \psi(\theta)-\beta(\theta) \psi(\theta) .
$$

Proof. Each of the separate terms of Eq. (3.12) is differentiable on $\mathscr{D}_{2}$ and leaves $\mathscr{D}_{2}$ invariant. By Lemma (3.6), $V_{t}$ is differentiable on $\mathscr{D}_{2}$ and the derivative may be calculated in the standard manner. Using Eq. (3.32) this yields at $t=0$

$$
(-i \hat{K} \psi)(\theta)=i \frac{\partial \alpha}{\partial t}(\theta, 0) \psi(\theta)+i \Phi\left\{\frac{\partial f}{\partial t}(\theta, 0)\right\} \psi(\theta)-i H_{0} \psi(\theta)-\omega \frac{\partial \psi}{\partial \theta}
$$

which gives Eq. (3.38) on simplification. The above calculation shows that $\mathscr{D}_{2} \cong \mathscr{D}(\hat{K})$ and since $\mathscr{D}_{2}$ is invariant under $\exp [-i \hat{K} t], \hat{K}$ is essentially self-adjoint on $\mathscr{D}_{2}$ by [20].

Theorem 3.8. The operator

$$
(\hat{K} \psi)(\theta)=-i \omega \frac{\partial \psi}{\partial \theta}+H_{0} \psi(\theta)+2^{\frac{1}{2}} \lambda \beta \Phi\left(e^{-i \theta} f\right) \psi(\theta)
$$

is essentially self-adjoint on $\mathscr{D}_{2}$. The unitary group

where

$$
\{\exp [-i \hat{K} t] \psi\}(\theta)=e^{i \alpha(t)} W\{f(\theta, t)\} e^{-i H_{0} t} \psi(\theta-\omega t)
$$

$$
f(\theta, t)=-2^{\frac{1}{2}} \lambda \beta e^{-i \theta} \int_{u=0}^{t} e^{i(\omega-S) u} b d u .
$$

Proof. We apply Lemma (3.7) with $\beta \equiv 0$ and

$$
g(\theta)=-2^{\frac{1}{2}} \lambda \beta e^{-i \theta} b .
$$

Then the cocycle $f$ is derived from Eq. (3.23). It is not difficult to show that $f, \alpha$ have continuous first partial derivatives and that $f(\theta, t) \in \mathscr{D}(S)$ for all $\theta, t$. It follows from Eq. (3.41) and Eq. (3.19) that $\alpha$ is actually independent of $\theta$.

Having given a rigorous meaning to $\hat{K}$ we now reverse Eq. (3.5) and define the self-adjoint operator $K$ on $\mathscr{K}$ by

$$
K=U \hat{K} U^{*} \text {. }
$$

We identify $\mathscr{K}$ with the space $l^{2}[\mathbb{Z}, \mathscr{F}]$ of all $\mathscr{F}$-valued square-summable sequences and define $\mathscr{D}_{3} \subseteq l^{2}[\mathbb{Z}, \mathscr{F}]$ as the space of all sequences of finite support with values in $\mathscr{F}_{\infty} \cap \mathscr{D}\left(H_{0}\right)$.

Lemma 3.9. $K$ is essentially self-adjoint on $\mathscr{D}_{3}$ and is given on that domain by

$$
K=\omega B_{3}+H_{0}+\lambda\left\{B_{-} a^{*}(b)+B_{+} a(b)\right\} .
$$


Proof. We define $\mathscr{D}_{4} \cong \hat{K}$ as the space of $C^{\infty}$ periodic functions from $[-\pi, \pi]$ to $\mathscr{F}_{\infty}$. Since $\hat{K}$ is essentially self-adjoint on $\mathscr{D}_{2}=\mathscr{D}_{1} \cap \mathscr{D}\left(H_{0}\right)$ it is also essentially self-adjoint on $\mathscr{D}_{4} \cap \mathscr{D}\left(H_{0}\right)$; this makes use of Eq. (3.39). Therefore $K$ is essentially self-adjoint on $\mathscr{D}_{5} \cap \mathscr{D}\left(H_{0}\right)$ where $\mathscr{D}_{5}=U \mathscr{D}_{4}$ is the space of all sequences $\psi \in l^{2}[\mathbb{Z}, \mathscr{F}]$ such that

$$
\sum_{n=-\infty}^{\infty}|n|^{2 k}\|\psi\|_{\alpha}^{2}<\infty
$$

for all $k=1,2,3, \ldots$ and all $\alpha>0$. It follows from Eq. (3.44) that $K$ is also essentially self-adjoint on $\mathscr{D}_{3}$.

We can now return to describe the sense in which the sequence of self-adjoint operators $K_{n}$ of Eq. (2.28) converge to $K$.

Theorem 3.10. If

$$
a_{n}=p-\frac{m}{2}-\frac{1}{2}
$$

then $\left(K_{n}-a_{n} 1\right)$ converges to $K$ in the strong generalised sense. Therefore for all $t \in \mathbb{R}$ and all $\psi \in \mathscr{K}$

$$
\lim _{n \rightarrow \infty} e^{-i\left(K_{n}-a_{n} 1\right) t} \psi=e^{-i K t} \psi,
$$

the limit being a norm limit.

Proof. It is clear that $\mathscr{D}_{3} \subseteq \mathscr{D}\left(K_{n}\right)$ for all $n$ and that

$$
\lim _{n \rightarrow \infty}\left(K_{n}-a_{n} 1\right) \psi=K \psi
$$

for all $\psi \in \mathscr{D}_{3}$. Since $K$ is essentially self-adjoint on $\mathscr{D}_{3}$ we have generalized strong convergence by [13, p. 429]. Strong convergence of the unitary groups is then a consequence $[5,13]$.

\section{§4. Time Evolution of the Field}

The time evolution of the field is given for finite $n$ by Eq. (2.23). The limiting behaviour of this for large $n$ is determined from Eq. (3.40) and Eq. (3.47). We work entirely in the space $\hat{\mathscr{K}}$ and first give a formula for the partial trace operation

$$
M: \mathscr{T}_{s}(\hat{\mathscr{K}}) \rightarrow \mathscr{T}_{s}(\mathscr{F}) .
$$

Lemma 4.1. If $\psi \in \hat{\mathscr{K}}$ then

$$
M(\psi \otimes \bar{\psi})=\int_{\theta=0}^{2 \pi} \psi(\theta) \otimes \psi(\theta)^{-} d \theta .
$$

Proof. The element $\varrho=M(\psi \otimes \bar{\psi})$ of $\mathscr{T}_{s}(\mathscr{F})$ is the solution of the equation

$$
\operatorname{tr}[\varrho A]=\langle(1 \otimes A) \psi, \psi\rangle
$$

where $A$ is an arbitrary bounded operator on $\mathscr{F}$. 
Then

$$
\begin{aligned}
\operatorname{tr}[\varrho A] & =\int_{0}^{2 \pi}\langle A \psi(\theta), \psi(\theta)\rangle d \theta \\
& =\int_{0}^{2 \pi} \operatorname{tr}\left[A\left\{\psi(\theta) \otimes \psi(\theta)^{-}\right\}\right] d \theta \\
& =\operatorname{tr}\left[A \int_{0}^{2 \pi}\left\{\psi(\theta) \otimes \psi(\theta)^{-}\right\} d \theta\right] .
\end{aligned}
$$

Note that putting $A=1$ gives

$$
\begin{aligned}
\operatorname{tr}[M(\psi \otimes \bar{\psi})] & =\int_{0}^{2 \pi} \operatorname{tr}\left[\psi(\theta) \otimes \psi(\theta)^{-}\right] d \theta \\
& =\int_{0}^{2 \pi}\|\psi(\theta)\|^{2} d \theta \\
& =\|\psi\|^{2}=\operatorname{tr}\left[\psi \otimes \psi^{-}\right],
\end{aligned}
$$

from which it follows by linearity that

for all $\varrho \in \mathscr{T}_{s}(\hat{\mathscr{K}})$.

$$
\operatorname{tr}[M(\varrho)]=\operatorname{tr}[\varrho]
$$

We can now prove the main theorem of the paper.

Theorem 4.2. For all $\varrho \in \mathscr{T}_{s}(\mathscr{F})$ and all $t \geqq 0$

$$
\lim _{n \rightarrow \infty} T_{n, t}(\varrho)=T_{t}(\varrho)
$$

the limit being taken in the trace norm, where

$$
T_{t}(\varrho)=\frac{1}{2 \pi} \int_{\theta=0}^{2 \pi} W\{f(\theta, t)\} e^{-i H_{0} t} \varrho e^{i H_{0} t} W\{f(\theta, t)\}^{*} d \theta
$$

and $f(\theta, t)$ is given by Eq. (3.41).

Proof. Since $M$ is a linear contraction it is sufficient to prove the theorem in the case where $\varrho$ is a vector state, say $\varrho=\varphi \otimes \varphi^{-}$for some unit vector $\varphi \in \mathscr{F}$. Then

$$
\begin{aligned}
\lim _{n \rightarrow \infty} \| & T_{n, t}(\varrho)-T_{t}(\varrho) \|_{\mathrm{tr}} \\
= & \lim _{n \rightarrow \infty}\left\|M\left(e^{-i K_{n} t} \varrho_{0} \otimes \varrho e^{i K_{n} t}\right)-M\left(e^{-i K t} \varrho_{0} \otimes \varrho e^{i K t}\right)\right\|_{\mathrm{tr}} \\
\leqq & \lim _{n \rightarrow \infty}\left\|e^{-i\left(K_{n}-a_{n} 1\right) t} \varrho_{0} \otimes \varrho e^{i\left(K_{n}-a_{n}\right) t}-e^{-i K t} \varrho_{0} \otimes \varrho e^{i K t}\right\|_{\mathrm{tr}} \\
= & \lim _{n \rightarrow \infty} \|\left\{e^{-i\left(K_{n}-a_{n} 1\right) t} e_{0} \otimes \varphi\right\} \otimes\left\{e^{-i\left(K_{n}-a_{n} 1\right) t} e_{0} \otimes \varphi\right\}^{-} \\
& -\left\{e^{-i K t} e_{0} \otimes \varphi\right\} \otimes\left\{e^{-i K t} e_{0} \otimes \varphi\right\}^{-} \|_{\mathrm{tr}} \\
\leqq & \lim _{n \rightarrow \infty} 2\left\|e^{-i\left(K_{n}-a_{n} 1\right) t}\left(e_{0} \otimes \varphi\right)-e^{-i K t}\left(e_{0} \otimes \varphi\right)\right\| \\
= & 0
\end{aligned}
$$

by Theorem (3.10). 
We now describe the connection of the above result with the theory of coherent states, introduced into quantum optics by Glauber $[9,14]$. We recall the decomposition

$$
\mathscr{F}=\mathbb{C} \oplus \mathscr{H} \oplus\left\{\otimes_{\text {sym }}^{2} \mathscr{H}\right\} \oplus\left\{\otimes_{\text {sym }}^{3} \mathscr{H}\right\} \oplus \cdots .
$$

For any $\psi \in \mathscr{H}$ we define the unit vector $\tilde{\psi} \in \mathscr{F}$ by

$$
\{\tilde{\psi}\}_{n}=\exp \left[-\|\psi\|^{2} / 2\right](n !)^{-\frac{1}{2}} \otimes^{n} \psi
$$

and the vacuum vector $\Omega$ by

$$
\Omega=\tilde{0}=1 \oplus 0 \oplus 0 \oplus \cdots .
$$

The coherent states are related to the free Hamiltonian and the Weyl operators by

See $[3,14]$.

$$
\begin{aligned}
e^{-i H_{0} t} \psi^{\sim} & =\left\{e^{-i S t} \psi\right\}^{\sim}, \\
W(f) \Omega & =\left\{i 2^{-\frac{1}{2}} f\right\}^{\sim} .
\end{aligned}
$$

Theorem 4.3. If the field is in the vacuum state at time zero then at time $t>0$ it is in a random phase, coherent state and the expected number of particles emitted up to time $t$ is

where

$$
\operatorname{tr}\left[T_{t}(\Omega \otimes \bar{\Omega}) N\right]=\lambda^{2}\left(\gamma-\frac{1}{2}+\frac{\mu}{2}\right)\left(-\gamma+\frac{1}{2}+\frac{\mu}{2}\right)\|h(t)\|^{2}
$$

$$
h(t)=\int_{u=0}^{t} e^{i(\omega-S) u} b d u .
$$

Proof. The state at time $t$ is

$$
\begin{aligned}
T_{t}(\Omega \otimes \bar{\Omega}) & =\frac{1}{2 \pi} \int_{\theta=0}^{2 \pi}\left[W\{f(\theta, t)\} e^{-i H_{0} t} \Omega\right] \otimes\left[W\{f(\theta, t)\} e^{-i H_{0} t} \Omega\right]^{-} d \theta \\
& =\frac{1}{2 \pi} \int_{\theta=0}^{2 \pi}[W\{f(\theta, t)\} \Omega] \otimes[W\{f(\theta, t)\} \Omega]^{-} d \theta \\
& =\frac{1}{2 \pi} \int_{\theta=0}^{2 \pi}\left\{i 2^{-\frac{1}{2}} f(\theta, t)\right\}^{\sim} \otimes\left\{i 2^{-\frac{1}{2}} f(\theta, t)\right\}^{--} d \theta \\
& =\frac{1}{2 \pi} \int_{\theta=0}^{2 \pi}\left\{i \lambda \beta e^{-i \theta} h(t)\right\}^{\sim} \otimes\left\{i \lambda \beta e^{-i \theta} h(t)\right\}^{--} d \theta
\end{aligned}
$$

which is what is called a random phase coherent state [9]. The formula (4.15) for the expected number of particles follows from

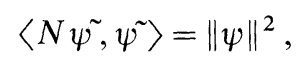

which is obtained by an easy calculation from Eq. (4.11). 
We should like the number of particles emitted to increase linearly with $t$. We first show that this is impossible if the radiation field consists of a single mode.

Theorem 4.4. Suppose that the quantum field consists of a single mode with energy $\omega_{1}$ per particle. Then the number of particles emitted up to time $t$ remains bounded if $\omega \neq \omega_{1}$ and increases with the square of $t$ if $\omega=\omega_{1}$.

Proof. The Hilbert space $\mathscr{H}$ is now one-dimensional and if $\omega \neq \omega_{1}$ we obtain from Eq. (4.16)

$$
h(t)=\left(e^{i\left(\omega-\omega_{1}\right) t}-1\right) b / i\left(\omega-\omega_{1}\right),
$$

which remains bounded, while if $\omega=\omega_{1}$,

so

$$
h(t)=b t
$$

$$
\operatorname{tr}\left[T_{t}(\Omega \otimes \bar{\Omega}) N\right]=\lambda^{2}\left(\gamma-\frac{1}{2}+\frac{\mu}{2}\right)\left(-\gamma+\frac{1}{2}+\frac{\mu}{2}\right)|b|^{2} t^{2} .
$$

We overcome this difficulty by the standard procedure of assuming that the quantum field has a continuum of energy levels [16, p. 734]. We suppose, precisely, that the single particle space $\mathscr{H}$ is infinitedimensional and that the Hamiltonian $S$ on $\mathscr{H}$ has absolutely continuous spectrum equal to $(0, \infty)$; for simplicity we also suppose that the spectrum is multiplicity free. All these assumptions, except the last, are satisfied for the quantised electromagnetic field. We also suppose the energy $\omega$ of the atomic transition is positive, $\omega>0$.

Lemma 4.5. There is a unitary isomorphism of $\mathscr{H}$ with $L^{2}(0, \infty)$ under which $S$ becomes the self-adjoint operator

$$
(S \psi)(x)=x \psi(x) .
$$

The element $h_{t}$ of $L^{2}(0, \infty)$ is given by

$$
h_{t}(x)=-i b(x)\left\{e^{i(\omega-x) t}-1\right\} /(\omega-x) .
$$

Proof. This is a straightforward consequence of spectral theory.

Taken in conjunction with Theorem (4.3) the following theorem shows that if the quantum field has a continuum of energy levels, then a linear rate of increase in the number of particles emitted is the normal occurrence.

Theorem 4.6. Let b be a continuously differentiable function in $L^{2}(0, \infty)$. Then as $t \rightarrow \infty$

$$
\left\|h_{t}\right\|^{2}=2 \pi|b(\omega)|^{2} t+O\left(t^{\frac{1}{2}}\right) .
$$


Proof. For technical reasons we calculate in the larger space $L^{2}(\mathbb{R})$, supposing $b(x)=0$ for $x \leqq 0$. We define

$$
\psi_{0}(x)=(2 \pi)^{-\frac{1}{2}} \int_{0}^{1} e^{-i x u} d u=(2 \pi)^{-\frac{1}{2}}\left(e^{-i x}-1\right) / i x
$$

so that $\psi_{0}(0)=(2 \pi)^{-\frac{1}{2}},\left\|\psi_{0}\right\|=1$ and $\psi_{0}$ is continuously differentiable. We also define

$$
\psi_{1}(x)=b(x+\omega)-(2 \pi)^{\frac{1}{2}} b(\omega) \psi_{0}(x)
$$

so that $\psi_{1}$ is continuously differentiable, $\psi_{1}(0)=0$ and

$$
\begin{aligned}
h_{t}(x+\omega)= & i \psi_{1}(x)\left\{e^{i x t}-1\right\} / x \\
& +i(2 \pi)^{\frac{1}{2}} b(\omega) \psi_{0}(x)\left\{e^{i x t}-1\right\} / x .
\end{aligned}
$$

We estimate the norms of the two parts of the right hand side separately.

$$
\begin{aligned}
& \int_{-\infty}^{\infty}\left|\psi_{1}(x)\left\{e^{-i x t}-1\right\} / x\right|^{2} d x \\
& \leqq 4 \int_{-\infty}^{\infty}\left|\psi_{1}(x) / x\right|^{2} d x<\infty
\end{aligned}
$$

since $\psi_{1}(0)=0$, this giving a bound independent of $t$. Also

$$
\begin{aligned}
\psi_{0}(x) & \left\{e^{-i x t}-1\right\} /(-i x) \\
& =\int_{s=0}^{t} e^{-i x s} \psi_{0}(x) d s \\
& =(2 \pi)^{-\frac{1}{2}} \int_{s=0}^{t} \int_{u=0}^{1} e^{-i x s-i x u} d u d s \\
& =(2 \pi)^{-\frac{1}{2}} \int_{s=0}^{t} \int_{u=-\infty}^{\infty} \chi_{[0,1]}(u) e^{-i x(s+u)} d s d u \\
& =(2 \pi)^{-\frac{1}{2}} \int_{u=-\infty}^{\infty}\left\{\int_{s=0}^{t} \chi_{[0,1]}(u-s) d s\right\} e^{-i x u} d u \\
& =(2 \pi)^{-\frac{1}{2}} \int_{u=-\infty}^{\infty} \varphi_{t}(u) e^{-i x u} d u
\end{aligned}
$$

where

$$
\varphi_{t}(x)=\left\{\begin{array}{ccl}
0 & \text { if } & x \leqq 0 \\
x & \text { if } & 0 \leqq x \leqq 1 \\
1 & \text { if } & 1 \leqq x \leqq t \\
t+1-x & \text { if } & t \leqq x \leqq t+1 \\
0 & \text { if } & x \geqq t+1
\end{array}\right.
$$




\section{Therefore}

$$
\begin{aligned}
& \int_{-\infty}^{\infty}\left|\psi_{0}(x)\left\{e^{-i x t}-1\right\} / x\right|^{2} d x \\
= & \int_{-\infty}^{\infty}\left|\varphi_{t}(x)\right|^{2} d x=t+O(1) .
\end{aligned}
$$

Putting these results together gives

$$
\left\|h_{t}\right\|=(2 \pi)^{\frac{1}{2}}|b(\omega)| t^{\frac{1}{2}}+O(1)
$$

from which Eq. (4.24) follows.

Acknowledgements. The author would like to thank J. T. Cannon for some helpful discussions.

\section{References}

1. Araki, H.: Hamiltonian formalism and the C.C.R's in quantum field theory. J. Math. Phys. 1, $492-504$ (1960)

2. Araki,H., Woods, E. J.: Representations of the C.C.R.'s for a non-relativistic infinite free Bose gas. J. Math. Phys. 4, 637-662 (1963)

3. Bargmann, V.: On a Hilbert space of analytic functions on an associated integral transform. Part 1. Commun. Pure Applied Math. 14, 187-214 (1961)

4. Cook, J. M.: The mathematics of second quantisation. Trans. Amer. Math. Soc. 74, $222-245(1953)$

5. Davies, E. B.: A generalisation of Kaplansky's theorem. J. London Math. Soc. 4, $435-436$ (1972)

6. Dicke, R. H.: Coherence in spontaneous radiation processes. Phys. Rev. 93, 99-110 (1954)

7. Emch, G.G., Wolfe, J.C.: A model for dissipative behaviour in non-linear quantum optics. J. Math. Phys. 13, 1236-1243 (1972)

8. Fain, V.M., Khanin, Ya.I.: Quantum electronics. Vol. 1. London: Pergamon Press 1969

9. Glauber, R.J.: Optical coherence and photon statistics. p. 63-186. Les Houches summer school of theoretical physics 1964, de Witt,C., Blandin, A., CohenTannoudji, C. (Eds.). New York: Gordon and Breach 1965

10. Hepp, K., Lieb, E. H.: On the superradiant phase transition for molecules in a quantised radiation field: the Dicke maser model. To appear.

11. Hermann, R.: Analytic continuation of group representations II. Commun. math. Phys. 3, 53-74 (1966)

12. Hermann, R.: Lie groups for physicists. New York: W. A. Benjamin Inc. 1966

13. Kato,T.: Perturbation theory for linear operators. Berlin-Heidelberg-New York: Springer 1966

14. Klauder,J.R., Sudarshan,E.C.G.: Fundamentals of quantum optics. New York: W. A. Benjamin Inc. 1968

15. Louisell, W.: Radiation and noise in quantum electronics. New York: McGraw Hill 1964

16. Messiah, A.: Quantum mechanics, Vol. 2. Amsterdam: North-Holland Publ. Co. 1966 
17. Parthasarathy, K.R., Schmidt,K.: Positive definite kernels, continuous tensor products, and central limit theorems of probability theory. Lecture notes BerlinHeidelberg-New York: Springer 1972

18. Scharf, G.: On a quantum mechanical maser model. Helv. Phys. Acta 43, 806-828 (1970)

19. Segal,I.E.: Foundation of the theory of dynamical systems of infinitely many degrees of freedom I. Mat.-Fys. Medd. Danske Vid. Selsk. 31 (12) 39 (1959)

20. Shale, D., Stinespring, W.F.: A note on the scattering of Boson fields. J. Math. Phys. 3, 915-921 (1962)

21. Tavis, M., Cummings, F.W.: Exact solution for an N-molecule-radiation-field Hamiltonian. Phys. Rev. 170, 379-384 (1968)

\author{
E. B. Davies \\ Mathematical Institute \\ 24-29 St. Giles \\ Oxford, England
}


